Open Access

\title{
Assessment of arterial stiffness using pulse wave velocity in tacrolimus users the first year post kidney transplantation: a prospective cohort study
}

Kelly Ann Birdwell ${ }^{1 *}$, Gilad Jaffe ${ }^{2}$, Aihua Bian ${ }^{3}$, Pingsheng $\mathrm{Wu}^{3}$ and Talat Alp Ikizler ${ }^{1}$

\begin{abstract}
Background: The leading cause of death in end stage renal disease is cardiovascular disease (CVD). Kidney transplantation is associated with improved survival over dialysis. We hypothesized that arterial stiffness, a marker of CVD, would improve in patients post kidney transplant, potentially explaining one mechanism of survival benefit from transplant.

Methods: After obtaining Institutional Review Board approval and informed consent, we performed a longitudinal prospective cohort study of 66 newly transplanted adult kidney transplant recipients, using aortic pulse wave velocity (PW) to assess arterial stiffness over a 12 month period. All patients were assessed within one month of transplant (baseline) and 12 months post transplant. The primary outcome was change in PW score at 12 months which we assessed using Wilcoxon Signed Rank test. Secondary analyses included correlation of predictors with PWV score at both time points.

Results: The median age of the cohort was 49.7 years at transplant, with $27 \%$ Black and $27 \%$ female. At baseline, $43 \%$ had tobacco use, $30 \%$ had a history of CVD, and $42 \%$ had diabetes. Median baseline calcium was $9.1 \mathrm{mg} / \mathrm{dL}$ and median phosphorus was $5.1 \mathrm{mg} / \mathrm{dL}$. Median PWV score was 9.25 and $8.97 \mathrm{~m} / \mathrm{s}$ at baseline versus month 12, respectively, showing no significant change (median change of $-0.07, p=0.7$ ). In multivariable regression, subjects with increased age at transplant $(p=0.008)$, diabetes $(p=0.002)$, and a higher baseline PW score $(p<0.001)$ were at increased risk of having a high PW score 12 months post transplant.

Conclusion: Aortic arterial stiffness does not progress in the first year post kidney transplant. Increasing age, diabetes, and higher baseline PWV score identify patients at risk for increased arterial stiffness. Further research that assesses patients for greater than one year and includes a control dialysis group would be helpful in further understanding the change in arterial stiffness post transplantation.
\end{abstract}

Keywords: Transplant, Cardiovascular, Outcome, Diabetes, Arterial stiffness

\footnotetext{
* Correspondence: kelly.birdwell@vanderbilt.edu

'Division of Nephrology and Hypertension, Vanderbilt University Medical

Center, 1161 21st Avenue, S-3223 MCN, Nashville, TN 37232, USA

Full list of author information is available at the end of the article
} 


\section{Background}

Cardiovascular disease (CVD) exerts a heavy burden in patients with chronic kidney disease (CKD), and it is the leading cause of death in the end stage renal disease (ESRD) population [1-3]. Contributors to CVD in ESRD include vascular arterial calcifications from abnormal mineral metabolism and inflammation related to poor kidney function. Kidney transplantation leads to improved survival compared to wait-listed dialysis patients, making it the renal replacement therapy of choice in eligible patients [4]. The reasons for this survival advantage are likely multifactorial, but include improved cardiovascular risk as a result of return of kidney function [5-7]. Even so, kidney transplant recipients continue to have a high burden of CVD, with CVD being the number one cause of death with a functioning transplant [1].

Increased aortic arterial stiffness is strongly associated with the development of CVD and is an independent predictor of cardiac and all-cause mortality [8]. Aortic pulse wave velocity (PWV) provides a non-invasive method to assess aortic arterial stiffness [8, 9]. Though often used in the research setting, several studies support using PWV in the clinic based on its reported associations with cardiovascular risk and all-cause mortality in a variety of patient populations, with one metaanalysis showing each increase of $1 \mathrm{~m} / \mathrm{s}$ in aortic PWV being associated with a $15 \%$ risk-adjusted increase in both cardiovascular and all-cause mortality [10]. Similar findings have been demonstrated specifically in patients with CKD and on maintenance dialysis, with PWV increasing over time in maintenance dialysis patients [11-14]. Higher aortic PWV has also been associated with all-cause mortality and cardiovascular events in few studies of prevalent kidney transplant recipients, but less is known about changes that may occur in arterial stiffness over time after successful kidney transplantation $[15,16]$.

We hypothesized that aortic arterial stiffness would be improved in patients receiving a kidney transplant, potentially explaining one mechanism of survival benefit from transplant. In this study we examined a prospective cohort of 66 kidney transplant recipients with aortic PWV measurements and assessed the changes in aortic arterial stiffness over the first year post kidney transplant.

\section{Methods}

\section{Study design}

All study procedures were approved by the Institutional Review Board (IRB) of Vanderbilt University Medical Center (VUMC). Informed consent was obtained from all study participants. We conducted a single-center, prospective cohort study of 66 newly transplanted adult kidney transplant recipients, using pulse wave velocity (PWV) to assess arterial stiffness over a 12 month period. Kidney transplant recipients were consecutively recruited from the VUMC Renal Transplant Clinic from August 2009 through September 2012. Inclusion criteria included patients aged $\geq 18$ who were undergoing or had recently undergone kidney transplantation. Participants were excluded if they were unwilling to participate or had atrial fibrillation, since this condition makes pulse wave velocity testing uninterpretable. Induction therapy was alemtuzumab with methylprednisolone in $85 \%$ of patients. All patients were maintained on tacrolimus and mycophenolate. Maintenance steroids were used in $9 \%$. Tacrolimus was dosed to a $12 \mathrm{~h}$ trough blood concentration of $8-10 \mathrm{ng} / \mathrm{ml}$ the first 6 months, then of $6-8 \mathrm{mg} / \mathrm{ml}$ for months 6 through 12 .

The study was composed of two visits, with patients assessed within 1 month of transplant (baseline) and 12 months post transplant. Demographic, medical, and social data was collected from each participant and confirmed in the electronic medical record. Participants underwent pulse wave velocity testing by a trained professional at the Vanderbilt Clinical Research Center using the SphygmoCor CPV Pulse Wave Velocity system (AtCor Medical, Sydney, Australia). Briefly stated, pulse wave velocity is the time it takes the arterial pressure wave to travel between two arterial sites, where PWV = distance (meters)/transit time (seconds). A higher PWV positively correlates with increased arterial stiffness. A more detailed description of pulse wave velocity procedure has been previously published [8]. In this study the carotid to femoral distance was selected, representing aortic arterial stiffness. The distance was measured by subtracting the distance between the carotid site and sternal notch from the distance between the sternal notch and the femoral site. For participants who were hemodialysis patients with vascular accesses, measures were taken on the non-access side. After resting $10 \mathrm{~min}$ in a supine position, hemodynamic measurements were obtained for each participant. All measurements were performed twice with the average of the two values used for analysis.

\section{Statistical analysis}

We expressed descriptive statistics as proportions for categorical variables and as means and standard deviations, or medians and interquartile ranges for continuous variables, depending on the distribution of the variables. The primary outcome was change in PWV score at 12 months from baseline, which we assessed using Wilcoxon Signed Rank test. We further assessed the change in PWV score stratified by subjects' diabetes status at baseline. Secondary analyses included predictors of PWV score at baseline and at 12 months post transplant. Spearman's rank correlation was used to test the association of age at baseline with PWV score at baseline and 12 months as well as the association of PWV score at baseline and at 12 months. We further 
conducted multivariable regression to determine predictors for both baseline and 12 month PWV measures. Age at transplantation, race, gender, diabetes status, and time in months on dialysis at baseline were included as potential predictors. Baseline PWV score was also included in the multivariable model to predict PWV score at 12 month post transplant. Normality of residuals of all linear models was diagnosed figuratively. We conducted all analyses using Rsoftware version $2 \cdot 11 \cdot 1$ (www.r-project.org) and used a two-sided $5 \%$ significance level for all statistical analyses.

\section{Results}

Seventy-eight kidney transplant recipients were enrolled into the study. Sixty-six of these had pulse wave velocity measures available at baseline and at 12 months post transplant. Characteristics of the study cohort are presented in Table 1 . The median age of the cohort was 49.7 years at transplant, with $27 \%$ black and $27 \%$ female. At baseline, $43 \%$ had tobacco use, $27 \%$ had a history of CVD, and $42 \%$ had diabetes. Mean blood pressure was $136 / 79 \mathrm{mmHg}$ at baseline and 131/77 $\mathrm{mmHg}$ at month 12 , with $94 \%$ of the cohort with pre-existing diagnosis of hypertension. Baseline calcium was $9.1 \mathrm{mg} / \mathrm{dL}$ and phosphorus was $5.1 \mathrm{mg} / \mathrm{dL}$. The median duration of dialysis prior to transplant was 1.74 years, though $18 \%$ of recipients had no dialysis time (pre-emptive transplant). At baseline, median serum creatinine was $8.4 \mathrm{mg} / \mathrm{dL}$ and estimated glomerular filtration rate (eGFR) was $10 \mathrm{~mL} / \mathrm{min} /$ $1.73 \mathrm{~m}^{2}$. Median serum creatinine 12 months post kidney transplant was $1.4 \mathrm{mg} / \mathrm{dL}$, corresponding to an eGFR $61 \mathrm{~mL} / \mathrm{min} / 1.73 \mathrm{~m}^{2}$.

The median PWV was $9.25 \mathrm{~m} / \mathrm{s}$ at baseline. At month 12 it was $8.97 \mathrm{~m} / \mathrm{s}$, showing no significant change in PWV over the study period (median change of -0.07 , $\mathrm{p}=0.7$; Fig. 1a). Twenty-five (37.9 \%) of the participants had a PWV at baseline greater than or equal to $10 \mathrm{~m} / \mathrm{s}$, indicating severe arterial stiffness at the time of transplant. Overall, about half the kidney transplant recipients showed an increase in PWV over 12 months, while the other half decreased, with no relation to PWV at baseline (Fig. 1b). Prior to analysis, we hypothesized diabetes would have an effect on PWV. Therefore we assessed the change in PWV in kidney transplant recipients with and without diabetes. As seen in Fig. 2, though patients without diabetes trended to a decrease in PWV and patients with diabetes an increase in PWV, these changes were not significant within or between the groups. Overall 8 new cardiovascular events were observed during the 12 month follow up period.

Our secondary analyses centered on predictors of PWV at baseline and at 12 months. Using Spearman correlation, age strongly correlated with pulse wave velocity
Table 1 Characteristics of the 66 kidney transplant recipients

\begin{tabular}{|c|c|}
\hline Characteristic & \\
\hline Age at transplant (years) & $49.7(41,61)$ \\
\hline \multicolumn{2}{|l|}{ Race } \\
\hline White & $46(70 \%)$ \\
\hline Black & $18(27 \%)$ \\
\hline Other & $2(3 \%)$ \\
\hline Sex (female) & $18(27 \%)$ \\
\hline \multicolumn{2}{|l|}{ Tobacco use } \\
\hline Never & $37(57 \%)$ \\
\hline Current & $5(8 \%)$ \\
\hline Former & $23(35 \%)$ \\
\hline Cardiovascular disease pre transplant ${ }^{a}$ & $18(27 \%)$ \\
\hline Diabetes pre transplant & $28(42 \%)$ \\
\hline \multicolumn{2}{|l|}{ Primary cause end stage renal disease } \\
\hline Diabetes & $21(32 \%)$ \\
\hline Hypertension & $13(20 \%)$ \\
\hline Glomerulonephritis & $11(17 \%)$ \\
\hline Cystic & $10(15 \%)$ \\
\hline Other & $11(17 \%)$ \\
\hline \multicolumn{2}{|l|}{ Serum creatinine (mg/dL) } \\
\hline Pre transplant & $8.4(6.2,9.7)$ \\
\hline 1 month post transplant & $1.5(1.2,1.7)$ \\
\hline 12 months post transplant & $1.4(1.2,1.6)$ \\
\hline \multicolumn{2}{|l|}{$\begin{array}{l}\text { Estimated glomerular filtration rate } \\
\left(\mathrm{mL} / \mathrm{min} / 1.73 \mathrm{~m}^{2}\right)\end{array}$} \\
\hline Pre transplant & $8.3(6,10.5)$ \\
\hline 1 month post transplant & $53.7(45.5,69.9)$ \\
\hline 2 months post transplant & $61(47.8,70.4)$ \\
\hline Calcium pre transplant (mg/dL) & $9.1(8.5,9.5)$ \\
\hline Phosphorus pre transplant (mg/dL) & $5.1(4.2,6.5)$ \\
\hline \multicolumn{2}{|l|}{ Systolic blood pressure $(\mathrm{mm} \mathrm{Hg})$} \\
\hline Baseline & $136.2(123,149)$ \\
\hline Month 12 & $131.3(120.5,143)$ \\
\hline \multicolumn{2}{|l|}{ Diastolic blood pressure $(\mathrm{mm} \mathrm{Hg})$} \\
\hline Baseline & $79(72,84)$ \\
\hline Month 12 & $77.1(42,83)$ \\
\hline Time on dialysis prior to transplant (days) & $636(362,1254)$ \\
\hline
\end{tabular}

Values expressed as median (25th, 75th percentiles) or number (percent) ${ }^{a}$ Cardiovascular disease includes coronary artery disease, stroke, and peripheral vascular disease

at baseline, showing increasing age with increasing baseline value $($ rho $=0.59, \mathrm{P}<.001)$. Neither time on dialysis nor the calcium-phosphorus product prior to transplant correlated with PWV at baseline. For PWV at 12 months, both PWV at baseline (rho $=0.87, \mathrm{p}<.001)$ and age $(\mathrm{rho}=0.61$, $\mathrm{P}<.001)$ were significantly correlated. No significant 


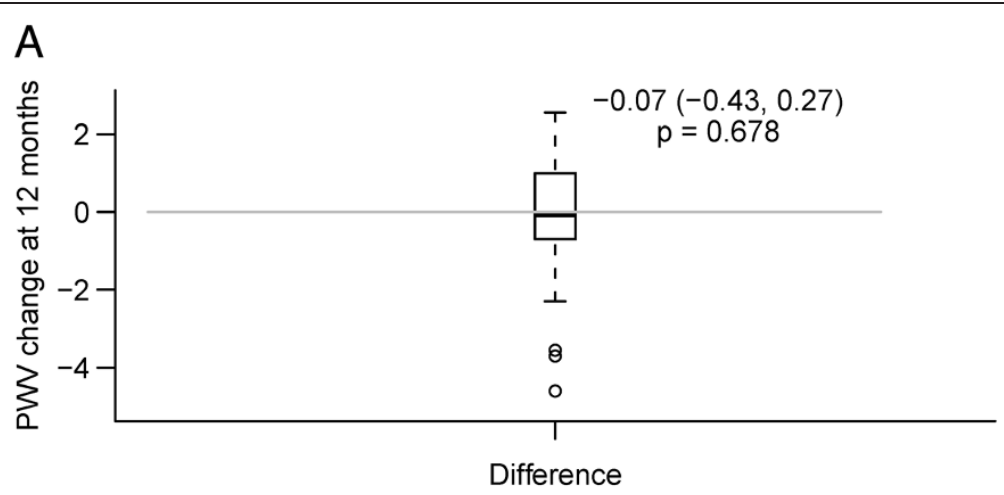

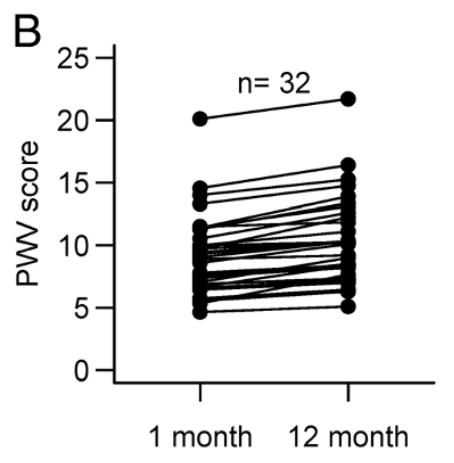

PWV change greater than and equal to 0

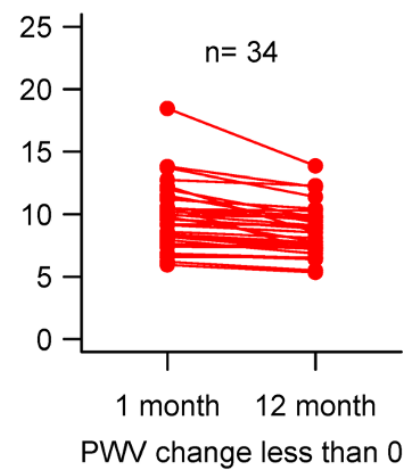

Fig. 1 a Box plot of change in PWV from baseline to month 12 in 66 kidney transplant recipients. $\mathbf{b}$ Individual line plots of PWV change from baseline to month 12 in each kidney transplant recipient, stratified by positive or negative change

correlation was seen between serum creatinine at 12 months and PWV at 12 months. Results for multivariable regression are shown in Table 2 . Increased age at transplant and presence of diabetes were significant predictors of higher PWV at baseline. Similarly, significant predictors of higher PWV at 12 months were increased

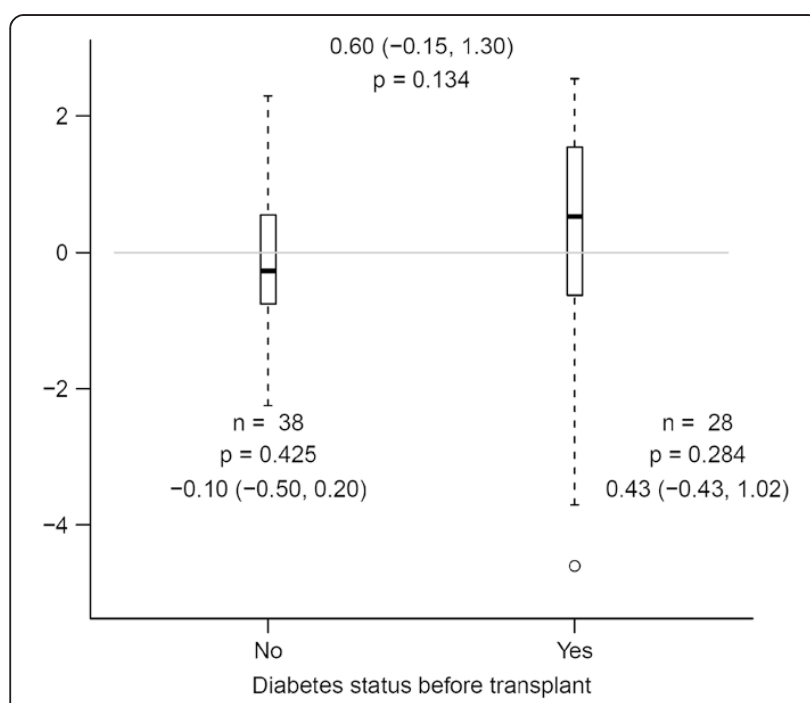

Fig. 2 Box plot of change in PW from baseline to month 12 in kidney transplant recipients with and without diabetes prior to transplant age and diabetes at time of transplant, as well as higher PWV at baseline.

\section{Discussion}

Patients who undergo kidney transplantation have improved survival and lower cardiovascular risk relative to wait-listed dialysis patients. This suggests that transplant may positively impact cardiovascular risk. Though this effect likely occurs through several processes, here we examined the effect of kidney transplantation on arterial stiffness by assessing PWV. In this prospective study of 66 new kidney transplant recipients, we showed that there was no change in aortic PWV over the first year post kidney transplant, suggesting arterial stiffness does not progress during this time period. Increasing age, having diabetes and higher baseline PWV score were associated with increased PWV score at 12 months and may help identify patients with increased arterial stiffness.

Previous studies have suggested that PWV progresses with time on dialysis. For example, Utescu and colleagues showed an annual increase of $0.84 \mathrm{~m} / \mathrm{s}$ per year (95\% CI $0.50-1.12 \mathrm{~m} / \mathrm{s}$ per year) in a cohort of 109 hemodialysis patients [12]. In another study of 88 waitlisted patients in which 39 were transplanted and 49 were not, during one year follow up no difference was 
Table 2 Multivariable linear regression model of PWV in kidney transplant recipients

\begin{tabular}{lcccl}
\hline Covariate & Effect & S.E. & $95 \% \mathrm{Cl}$ & $\mathrm{P}$ \\
\hline Predictors of PWV at baseline & & & & \\
Age at transplant & 2.8 & 0.5 & $1.8-3.8$ & $<0.0001$ \\
Non-white race & 0.4 & 0.6 & $-0.8-1.6$ & 0.5 \\
Female sex & 0.5 & 0.6 & $-0.7-1.7$ & 0.4 \\
Diabetes pre transplant & 2.5 & 0.6 & $1.4-3.6$ & $<0.0001$ \\
CVD pre transplant ${ }^{\mathrm{a}}$ & 0.9 & 0.6 & $-0.4-2.1$ & 0.2 \\
Time on dialysis $^{\text {Predictors of PWV at 12 months }}$ & 0.2 & 0.3 & $-0.3-0.7$ & 0.5 \\
PWV at baseline & 2 & 0.3 & $1.5-2.6$ & $<0.0001$ \\
Age at transplant & 1.1 & 0.4 & $0.3-1.9$ & 0.01 \\
Non-white race & 0.4 & 0.4 & $-0.4-1.2$ & 0.4 \\
Female sex & 0.5 & 0.4 & $-0.3-1.3$ & 0.2 \\
Diabetes pre transplant & 1.5 & 0.4 & $0.6-2.3$ & 0.001 \\
CVD pre transplant $^{\mathrm{a}}$ & 0.04 & 0.4 & $-0.8-0.9$ & 0.9 \\
Time on dialysis & 0.2 & 0.2 & $-0.2-0.5$ & 0.4 \\
\hline
\end{tabular}

${ }^{\mathrm{a}}$ Cardiovascular disease (CVD) includes coronary artery disease, stroke, and peripheral vascular disease

seen in PWV between the 2 groups, though follow up time for those transplanted was short (median 6.3 months) [17]. In our study, in contrast to dialysis patients, we did not see PWV progression over the first year post transplant. One interpretation could be that restoration of kidney function with transplant has a positive impact on slowing the process of arterial stiffness. However, further study is needed to see if this trend continues over longer time periods, and more importantly if this is associated with decreased CVD events. Given the adverse metabolic effects of routinely used immunosuppression drugs, it is possible that with more time the balance shifts the opposite direction.

For kidney transplant recipients, prior cross-sectional studies have shown that transplant recipients have higher aortic PWV measurements than historical healthy controls. The reported median normal values for a large European population of individuals aged 40-49 was $6.9 \mathrm{~m} / \mathrm{s}$ and aged 50-59 was $8.1 \mathrm{~m} / \mathrm{s}$ compared to $9.25 \mathrm{~m} / \mathrm{s}$ in our patient cohort with a median age of 49.7 years [9]. In a retrospective study, 106 patients in Lebanon at a mean 54.1 months post transplant had mean aortic PWV of $11.1 \pm 2.8 \mathrm{~m} / \mathrm{s}$ compared to $8.7 \pm 1.6 \mathrm{~m} / \mathrm{s}$ in a previously studied healthy male cohort. The mean PWV value in that study was much higher than what we observed in our cohort. In addition, the transplant recipients who had experienced more cardiovascular events at time of PWV assessment had a significantly higher aortic PWV than those who did not $(12.8 \pm 4.3$ vs $10.9 \pm 2.5 \mathrm{~m} / \mathrm{s}$, respectively, $\mathrm{p}<0.05)$ [18]. Similarly a study from Belgium of primarily white patients showed a higher baseline measure of PWV was associated with more cardiovascular events after a mean follow up of 5 years $(11.2 \mathrm{vs} 9.2 \mathrm{~m} / \mathrm{s}$, $\mathrm{p}<0.001)$ [19].

These prior studies had single time points of PWV assessments of prevalent transplant recipients, with patients enrolled at varying times post transplantation. Our study was different in that it followed incident patients from time of transplant, with measurements at baseline and one year, adding further information about what happens to PWV over time. Our results indicate that changes in aortic stiffness may be slowed with successful transplantation, consistent with two previous smaller studies over a shorter time period of 3 months [20,21]. In the study by Ignace, et al. aortic stiffness changes post transplantation were dependent on age, with improvement seen only in transplant recipients older than 50 years [21]. A previous study by Zoungas, et al. observed aortic PWV in patients over 1 year showing a slight improvement, but the majority of these patients were on cyclosporine [15]. Our study has similar findings but in tacrolimus treated patients, which is important since tacrolimus is the most commonly prescribed calcineurin inhibitor, used as the initial choice in $91 \%$ of patients in the United States [1]. To our knowledge this is the first study to report carotid PWV in a cohort entirely maintained on tacrolimus of this duration. A Japanese study of 58 patients on tacrolimus followed only up to 6 months post transplantation showed a decrease in brachial-ankle PWV in a low risk population of younger (mean age 40.5 years) kidney transplant recipients [22].

In contrast to our study, a study in Poland found that carotid PWV increased with time post transplantation, but these patients were already a median time of 36 months post transplantation at time of enrollment, differing from our population of newly transplanted patients. Similar PWV values to those in our study were observed in the 61 kidney transplant recipients with an eGFR of 55 $\mathrm{mL} / \mathrm{min} / 1.73 \mathrm{~m}^{2}$, where the initial measured carotid PWV was $9.1 \mathrm{~m} / \mathrm{s}$. In this group, however, PWV increased to $9.8 \mathrm{~m} / \mathrm{s}$ when measured 24 months later [23]. One possible explanation for this finding was the prolonged exposure to transplant immunosuppression. Indeed, some studies have examined the effects of different classes of immunosuppression on aortic stiffness. In a randomized study in France, low cardiovascular risk groups of newly transplanted kidney patients were maintained on cyclosporine or randomized to sirolimus at week 12 and followed for a variety of cardiovascular related outcomes, including carotid PWV, for 1 year. Those in the sirolimus group had significantly lower PWV at follow up [24]. Similarly, another study compared carotid PWV in kidney transplant recipients on belatacept versus calcineurin inhibitors, and found after 5 years post transplantation, 
more patients in the calcineurin inhibitor group had a PWV greater than $8.1 \mathrm{~m} / \mathrm{s}$ than in the belatacept group [25]. These studies support that calcineurin inhibitors may have more detrimental cardiovascular effects long term.

Strengths of our study included the prospective longitudinal design and duration of follow up, including the fact that all patients started follow up at time of their transplant, so they all had been exposed to transplant immunosuppression the same length of time. In addition, a large percent of the cohort was black, allowing study of a population not frequently available in other cohorts of kidney transplant recipients. A weakness was our inability to adjust for multiple covariates without overfitting the model due to our relatively small sample size, though we were able to adjust for all demographics and important cardiovascular risk factors like diabetes and pre-existing cardiovascular disease. Also due to the small number of cardiovascular events in this study current cohort, we were not able to test associations of PWV with these. In addition, overall our population was a low risk population for acute rejection and low risk for immediate cardiovascular events due to our selection process pre transplant.

\section{Conclusions}

In a prospective study of new kidney transplant recipients treated with tacrolimus, we showed that there was no change in aortic PWV over the first year post kidney transplantation, suggesting arterial stiffness does not progress during this time period in a cohort of low risk individuals for cardio-renal outcomes. Further prospective research regarding arterial stiffness after transplant would be helpful to better understand the impact of transplant on cardiovascular risk. Specifically studies with longer follow-up time and with adequate number of cardiovascular events are necessary. Ultimately this may help identify kidney transplant patients at higher risk for CVD events and to target for intervention.

\footnotetext{
Abbreviations

CKD: Chronic kidney disease; CVD: Cardiovascular disease; ESRD: End stage renal disease; PWV: Pulse wave velocity; VUMC: Vanderbilt University Medical
} Center; eGFR: Estimated glomerular filtration rate.

\section{Competing interests}

The authors declare that they have no competing interests.

\begin{abstract}
Authors' contributions
$\mathrm{KB}$ conceived and participated in design of the study, and drafted the manuscript. GJ participated in design of the study and helped with data acquisition. $A B$ participated in design of the study and helped perform the statistical analysis. PW participated in design of the study, performed the statistical analysis and helped to draft the manuscript. TI conceived the study, participated in design of the study, and helped to draft the manuscript. All authors read and approved the final manuscript.
\end{abstract}

\section{Acknowledgements}

Supported in part by Vanderbilt CTSA grant UL1TR000445 from the National Center for Advancing Translational Sciences and by Award Number S10RR027033 from the National Center for Research Resources. Other grant support includes K24 DK62849 (Ikizler) and P30 DK079341 from the National Institute of Diabetes, Digestive and Kidney Diseases; R01 HL070938 (Ikizler) from the National Heart, Lung, and Blood Institute; and K23 GM100183 (Birdwell) from the National Institute of General Medical Sciences. The content is solely the responsibility of the authors and does not necessarily represent the official views of the National Institutes of Health. General support provided by the Vanderbilt Center for Kidney Disease.

\section{Author details}

'Division of Nephrology and Hypertension, Vanderbilt University Medical Center, 1161 21st Avenue, S-3223 MCN, Nashville, TN 37232, USA. ${ }^{2}$ University at Buffalo School of Medicine and Biomedical Sciences, 3435 Main Street, Buffalo, NY 14260, USA. ${ }^{3}$ Department of Biostatistics, Vanderbilt University Medical Center, 2525 West End Avenue, Suite 11000, Nashville, TN 37203, USA.

Received: 3 February 2015 Accepted: 23 June 2015

Published online: 02 July 2015

\section{References}

1. 2013 USRDS Annual Data Report. Atlas of Chronic Kidney Disease and End-Stage Renal Disease in the United States. Am J Kidney Dis. 2014;63(1):e1-e478.

2. Levey AS. Controlling the epidemic of cardiovascular disease in chronic renal disease: where do we start? Am J Kidney Dis. 1998;32(5 Suppl 3):S5-13.

3. Go AS, Chertow GM, Fan D, McCulloch CE, Hsu CY. Chronic kidney disease and the risks of death, cardiovascular events, and hospitalization. N Engl J Med. 2004;351(13):1296-305.

4. Wolfe RA, Ashby VB, Milford EL, Ojo AO, Ettenger RE, Agodoa LY, et al. Comparison of mortality in all patients on dialysis, patients on dialysis awaiting transplantation, and recipients of a first cadaveric transplant. N Engl J Med. 1999;341(23):1725-30.

5. Hunsicker LG. A survival advantage for renal transplantation. N Engl J Med. 1999;341(23):1762-3.

6. Meier-Kriesche HU, Schold JD, Srinivas TR, Reed A, Kaplan B. Kidney transplantation halts cardiovascular disease progression in patients with end-stage renal disease. Am J Transplant. 2004;4(10):1662-8.

7. Meier-Kriesche HU, Ojo AO, Port FK, Arndorfer JA, Cibrik DM, Kaplan B. Survival improvement among patients with end-stage renal disease: trends over time for transplant recipients and wait-listed patients. J Am Soc Nephrol. 2001;12(6):1293-6.

8. Boutouyrie P, Fliser D, Goldsmith D, Covic A, Wiecek A, Ortiz A, et al. Assessment of arterial stiffness for clinical and epidemiological studies: methodological considerations for validation and entry into the European Renal and Cardiovascular Medicine registry. Nephrol Dial Transplant. 2014;29(2):232-9.

9. Reference Values for Arterial Stiffness' Collaboration. Determinants of pulse wave velocity in healthy people and in the presence of cardiovascular risk factors: 'establishing normal and reference values'. Eur Heart J. 2010;31(19):2338-50.

10. Vlachopoulos C, Aznaouridis K, Stefanadis C. Prediction of cardiovascular events and all-cause mortality with arterial stiffness: a systematic review and meta-analysis. J Am Coll Cardiol. 2010;55(13):1318-27.

11. Baumann M, Wassertheurer S, Suttmann Y, Burkhardt K, Heemann U. Aortic pulse wave velocity predicts mortality in chronic kidney disease stages $2-4$. J Hypertens. 2014;32(4):899-903.

12. Utescu MS, Couture V, Mac-Way F, De Serres SA, Marquis K, Lariviere R, et al. Determinants of progression of aortic stiffness in hemodialysis patients: a prospective longitudinal study. Hypertension. 2013;62(1):154-60.

13. Blacher J, Guerin AP, Pannier B, Marchais SJ, Safar ME, London GM. Impact of aortic stiffness on survival in end-stage renal disease. Circulation. 1999;99(18):2434-9.

14. Verbeke F, Van Biesen W, Honkanen E, Wikstrom B, Jensen PB, Krzesinski JM, et al. Prognostic value of aortic stiffness and calcification for cardiovascular events and mortality in dialysis patients: outcome of the calcification outcome in renal disease (CORD) study. Clin J Am Soc Nephrol. 2011;6(1):153-9.

15. Zoungas S, Kerr PG, Chadban S, Muske C, Ristevski S, Atkins RC, et al. Arterial function after successful renal transplantation. Kidney Int. 2004;65(5):1882-9. 
16. Mitchell A, Opazo Saez A, Kos M, Witzke O, Kribben A, Nurnberger J. Pulse wave velocity predicts mortality in renal transplant patients. Eur J Med Res. 2010;15(10):452-5.

17. Bachelet-Rousseau C, Kearney-Schwartz A, Frimat L, Fay R, Kessler M, Benetos A. Evolution of arterial stiffness after kidney transplantation. Nephrol Dial Transplant. 2011;26(10):3386-91.

18. Bahous SA, Stephan A, Barakat W, Blacher J, Asmar R, Safar ME. Aortic pulse wave velocity in renal transplant patients. Kidney Int. 2004;66(4):1486-92.

19. Verbeke F, Marechal C, Van Laecke S, Van Biesen W, Devuyst O, Van Bortel $L M$, et al. Aortic stiffness and central wave reflections predict outcome in renal transplant recipients. Hypertension. 2011;58(5):833-8.

20. Covic A, Goldsmith DJ, Gusbeth-Tatomir P, Buhaescu I, Covic M. Successful renal transplantation decreases aortic stiffness and increases vascular reactivity in dialysis patients. Transplantation. 2003;76(11):1573-7.

21. Ignace S, Utescu MS, De Serres SA, Marquis K, Gaudreault-Tremblay MM Lariviere $\mathrm{R}$, et al. Age-related and blood pressure-independent reduction in aortic stiffness after kidney transplantation. J Hypertens. 2011;29(1):130-6.

22. Hotta K, Harada H, Sasaki H, Iwami D, Fukuzawa N, Morita K, et al. Successful kidney transplantation ameliorates arterial stiffness in end-stage renal disease patients. Transplant Proc. 2012;44(3):684-6.

23. Strozecki P, Adamowicz A, Kozlowski M, Wlodarczyk Z, Manitius J. Progressive arterial stiffening in kidney transplant recipients. Ann Transplant. 2011:16(3):30-5.

24. Joannides R, Monteil C, de Ligny BH, Westeel PF, lacob M, Thervet E, et al. Immunosuppressant regimen based on sirolimus decreases aortic stiffness in renal transplant recipients in comparison to cyclosporine. Am J Transplant. 2011;11(11):2414-22.

25. Melilli E, Bestard-Matamoros O, Manonelles-Montero A, Sala-Bassa N, Mast R, Grinyo-Boira JM, et al. Arterial stiffness in kidney transplantation: a single center case-control study comparing belatacept versus calcineurin inhibitor immunosuppressive based regimen. Nefrologia. 2015;35(1):58-65.

\section{Submit your next manuscript to BioMed Central and take full advantage of:}

- Convenient online submission

- Thorough peer review

- No space constraints or color figure charges

- Immediate publication on acceptance

- Inclusion in PubMed, CAS, Scopus and Google Scholar

- Research which is freely available for redistribution 